\section{$\underset{\substack{\text { hommes } \\ \text { \& migrations }}}{ }$}

\section{Hommes \& migrations}

Revue française de référence sur les dynamiques

migratoires

$1311 \mid 2015$

Femmes et migrations

\title{
Les migrations féminines vers la Turquie de 1990 à nos jours
}

\section{Gökçe Bayindir Goularas, Nihan Kocaman et Ayşe Betül Nuhoğlu}

\section{(2) OpenEdition \\ Journals}

\section{Édition électronique}

URL : http://journals.openedition.org/hommesmigrations/3254

DOI : 10.4000/hommesmigrations.3254

ISSN : 2262-3353

\section{Éditeur}

Musée national de l'histoire de l'immigration

\section{Édition imprimée}

Date de publication : 1 juillet 2015

Pagination : 47-51

ISBN : 978-2-919040-32-2

ISSN : $1142-852 X$

\section{Référence électronique}

Gökçe Bayindir Goularas, Nihan Kocaman et Ayşe Betül Nuhoğlu, « Les migrations féminines vers la Turquie de 1990 à nos jours », Hommes \& migrations [En ligne], 1311 | 2015, mis en ligne le 01 juillet 2018, consulté le 19 avril 2019. URL : http://journals.openedition.org/hommesmigrations/3254 ; DOI : 10.4000/hommesmigrations.3254 


\title{
LES MIGRATIONS FÉMININES VERS LA TURQUIE DE 1990 JUSQU'À NOS JOURS
}

Par GÖKÇE BAYINDIR GOULARAS, maître de conférences, département francophone de science politique et relations internationales, université Yeditepe (Istanbul), chercheure associée, Géographie-Cités (UMR 8504), NIHAN KOCAMAN, étudiante en master francophone des relations internationales, université Yeditepe, et AYŞE BETÜL NUHOĞLU, étudiante en master francophone des relations internationales, université Yeditepe.

\author{
La Turquie est devenue un pays d'immigration à partir des \\ années 1990, notamment pour les femmes originaires des pays \\ de l'ex-URSS. Frappées par le chômage dans leur pays d'origine, \\ elles ont trouvé sur le marché du travail turc de nouveaux \\ débouchés dans les métiers du tourisme ou des services à \\ la personne. Mais, derrière l'attractivité économique de la Turquie, \\ encouragée par sa politique de visas, les femmes migrantes \\ originaires des pays de l'Est connaissent aussi, dans le secteur \\ informel, une précarité grandissante.
}

Dans les dernières décennies, les recherches sur les migrations internationales se sont intéressées à la féminisation de la migration ${ }^{1}$. Ces flux migratoires féminins ont touché également la Turquie, devenue rapidement un pays récepteur de femmes migrantes, en situation régulière ou irrégulière, et en provenance de l'Europe de l'Est, de la Russie et de certains pays de la région caucasienne. La Turquie a attiré vers elle ces migrantes par son marché ouvert à la main-d'œuvre étrangère, particulièrement dans certains secteurs réservés à une population migrante féminine ${ }^{2}$.

Avec la transformation socio-économique des pays socialistes, la plupart des anciennes travailleuses du secteur agricole, de l'industrie légère, des services sociaux et de l'éducation se sont retrouvées dans un environnement très compétitif occasionnant chômage, pauvreté et inégalités sociales ${ }^{3}$. La situation

1. Serpil Sancar (dir.), Birkaç Arpa Boyu. 21. Yüzyıla Girerken Türkiye'de Feminist Çalışmalar. Prof. Dr. Nermin Abadan Unatıa Armağan, Istanbul, Koç Üniversitesi Yayınları, 2011, p. 187. 2. Ici, il faut mentionner que, depuis le début des années 199o, à l'échelle mondiale, les femmes originaires de l'Europe de l'Est, de la Russie et de certains pays de la région caucasienne constituent une partie importante des migrantes féminines actuelles. Voir Saniye Dedeoğlu, “Türkiye’de Göçmenlerin Sosyal Dışlanması : İstanbul HazırGiyim Sanayinde Çalışan Azerbaycanlı Göçmen Kadınlar Örneği”, in Ankara Üniversitesi SBF Dergisi, vol. 1, n 66, 2011, p. 33. 3. Derya Demirdizen, "Türkiye'de Ev Hizmetlerinde Çalışan Göçmen Kadınlar: Yeni Düzenlemelerle Yarı Köle Emeğine Doğru mu?”, in Çalısma ve Toplum, vol. $38, n^{\circ} 3,2013$, p. 330. 
des femmes est devenue de plus en plus difficile, avec la suppression des aides sociales du régime socialiste qui allégeaient leur charge domestique et économique. Suite à la chute du régime soviétique et au passage rapide à l'économie de marché, ce qu'un certain nombre de chercheurs appellent "la féminisation du chômage et de la pauvreté" a provoqué la migration régulière et irrégulière de milliers de femmes vers d'autres pays offrant de meilleures opportunités économiques ${ }^{4}$.

Cette première vague de migration concernait particulièrement les femmes provenant de Moldavie, de Bulgarie, de Roumanie, d'Ukraine et de Russie. Au milieu des années 1990, elle a été suivie par une deuxième vague de femmes originaires de la Géorgie, d'Azerbaïdjan, d’Arménie, du Turkménistan et d'Ouzbékistan ${ }^{5}$.

\section{La Turquie, un pays attractif pour les migrantes internationales}

Depuis sa fondation en 1923, la Turquie se présente comme un pays d'origine, de transit et de destination des migrations. Jusqu'aux années 1960, la Turquie se caractérise par l'accueil de vagues de migrations des habitants musulmans des anciens territoires ottomans. Les mouvements migratoires de la Turquie vers l'étranger concernaient plutôt les minorités non musulmanes du pays. La période des années de 1960 et de 1970 est marquée par la migration des citoyens turcs vers les pays industrialisés de l'Europe ainsi que par le départ de réfugiés politiques lors des périodes de coups d'État. À partir des années 1980, tout en gardant son statut de pays d'émigration, notamment pour les Turcs de Bulgarie et, un peu plus tard, pour les musulmans de Bosnie, la Turquie est devenue un pays de transit pour les migrants et les réfugiés originaires des pays du Moyen-Orient et de l'Asie qui tentent d'accéder aux pays européens. De 1970 à 2000, la population étrangère a montré une augmentation de $576 \%$. Le pic de la migration se situe en 1990, avec une population étrangère de 245600 personnes contre 50642 enregistrées dans la décennie précédente. En 2013, la population étrangère en Turquie correspondait à 2,5\% de la population totale, avec environ 1870000 étrangers en situation régulière ${ }^{6}$. Ces chiffres n'incluent pas les migrants en situation irrégulière. Ainsi, il est difficile d'estimer le nombre exact et la provenance de la population étrangère vivant en Turquie ${ }^{7}$. Néanmoins, les données de la Direction de la sécurité du ministère turc des Affaires intérieures montrent qu'entre 2000 et 2010, parmi les personnes ayant migré pour des motifs économiques et qui ont été arrêtées par les forces de l'ordre turques, les ressortissants de Moldavie, de Géorgie, de Roumanie, de la Fédération russe et d'Ukraine représentaient $60 \%$ des migrants, sur un total de 225802 migrants ayant participé à la migration circulaire ${ }^{8}$. La répartition géographique de la population migrante dépend des origines géographiques de son pays d'origine et de son secteur d'emploi, mais aussi des régions les plus développées de la Turquie, là où se trouvent les principales grandes villes comme Istanbul, Ankara, Izmir et Bursa, qui accueillent presque les trois quarts des migrants internationaux. $^{9}$

\section{Du commerce de la valise à la migration irrégulière}

La migration des femmes originaires des pays del'exURSS vers la Turquie a débuté vers la fin des années 1980 par le "commerce de la valise" qui nécessitait de "faire la navette" entre le pays d'origine et la Turquie pour réaliser des activités commerciales

4. Serpil Sancar (dir.), op. cit., 2011, p.188. 5. Emel Akalın Ayşe, “Türkiye’de Göçmen Ev isşçilerinin Çalışma Koşulları, VI. Sosyal Insan Hakları Ulusal Sempozyumu”, in Petrol iş Yayını, n 119, 2014, p. 327. 6. Mustafa Yakar, Kadir Temurçin, "Yurtdışından Türkiyesye Kim, Nereye Göç Ediyor?", in Journal of World of Turks, vol. 5, n 1, 2013, pp. 219-220. 7. Ici, il faut rappeler que la Turquie est un pays signataire de la convention de Genève de 1951 relative au statut des réfugiés, avec une réserve géographique suivant laquelle elle ne peut accepter que les personnes provenant des pays de l'Europe en tant que réfugiés. 8. Ahmet Içduygu, Damla Aksel, "Irregular Migration in Turkey”, IOM Report, Ankara, 2012, p. 23. www.turkey.iom.int/documents/IrregularMigration/ IOM Report_11022013.pdf 9. Mustafa Yakar, Kadir Temurçin, op. cit., p. 220. 
à petite échelle ${ }^{10}$. Les migrantes, achetant des biens en Turquie pour les revendre dans leur pays, étaient souvent bien éduquées et pratiquaient ce type de commerce informel afin d'augmenter leurs revenus ${ }^{11}$. La difficulté de migrer vers les pays de l'Union européenne a, de son côté, accéléré les mouvements migratoires internationaux vers des pays qui facilitent l'accès des migrants à leur territoire, comme la Turquie. Au début des années 2000, environ 100000 personnes arrivaient chaque année. Par la suite, avec l'adhésion des pays de l'Europe de l'Est à l'Union européenne, le nombre de ces migrantes a diminué autour de 50000 personnes par an ${ }^{12}$. En revanche, une partie des migrantes qui participaient à la migration circulaire est restée illégalement à l'intérieur des frontières turques après la péremption de leur visa ${ }^{13}$. La plupart d'entre elles ont trouvé des opportunités économiques dans des secteurs où les femmes sont surreprésentées par rapport aux hommes, comme l'industrie manufacturière, l'agriculture, les métiers du tourisme et des loisirs, les services domestiques et le travail du $\operatorname{sexe}^{14}$. La connaissance d'une autre langue que le turc, le niveau d'éducation élevé mais surtout la faible rémunération des migrantes par rapport aux salariées locales ont favorisé leur emploi dans certains secteurs de l'économie informelle ${ }^{15}$.

\section{L'accélération de la migration féminine vers la Turquie}

À partir des années 2000, les mouvements migratoires vers la Turquie se sont accélérés ${ }^{16}$. Aujourd'hui, les ressortissantes de la Moldavie, de l'Ukraine, suivies de celles de la Roumanie et

Gulnur : "Ma carte de travail oügour m'a permis de m'inscrire à la fac à Paris. J'y ai glissé des photos, dont celle de ma mère au même âge que moi aujourd'hui." (c) Sophie Pasquet - Hans lucas.

de la Géorgie, sont majoritaires par rapport aux hommes originaires des autres pays de l'ex-URSS ${ }^{17}$. Les régimes de visas appliqués lors de la même période ont indirectement encouragé la migration irrégulière de main-d'œuvre. Les accords bilatéraux signés au début des années 1990 entre la Turquie et les pays de l'ex-URSS qui ont supprimé le visa d'entrée en Turquie ou facilité l'obtention du visa apparaissent comme un autre facteur d'attraction vers la Turquie. À partir de 1990, le nombre de ressortissants des pays de la Communauté des États indépendants (CEI) possédant un visa touristique a augmenté (de 43369 personnes en 1989 contre 223211 en 1990) et il atteint à son sommet en 1992 (1 241010 personnes) grâce, notamment, à la suppression du visa avec l'Azerbaïdjan, le Turkménistan et le Kazakhstan et plus tard avec les autres États de la $C E{ }^{18}$. La présence des réseaux de migrantes et des agences d'emploi a également

10. Taner Akpınar, “Türkiye’ye yönelik kaçak işgücü göçü”, in Ankara Üniversitesi SBF Dergisi, vol. 65, n 3, 2010, pp. 6-11. 11. Jean-François Pérouse, "Les formes de mobilités transcaucasiennes et balkaniques à Istanbul”, in Balkanologie, vol. X, $n^{\circ} 1-2$, 2008. 12. Ahmet Içduygu, Sema Erder, Faruk Gençkaya Ömer, “Türkiye'nin Uluslararası Göç Politikaları, 1923-2023: Ulus-devlet Oluşumundan Ulus-ötesi Dönüşümlere”, in MiReKoç Proje Raporları, n 1, Istanbul, 2014, p. 235. 13. Ibid. 14. Emel Akalın Ayşe, op. cit., p. 324. 15. Neslihan Atatimur, "Reasons and consequences of international labor migration of women into Turkey: Ankara Case", A thesis submitted to the graduate school of natural and applied sciences of Middle East technical University, for the degree of Master of Science in regional planning in city and regional planning, Ankara, 2008, p. 185. 16. Mine Eder, “Moldovalı Yeni Göçmenler Üzerinden Türkiye’deki Neoliberal Devleti Yeniden Düşünmek”, in Toplum ve Bilim, n 108 , 2007, p. 131. 17. Ibid. À titre d'exemple, suivant les données de 2000, la Turquie a reçu la migration de 612 femmes contre 109 hommes de Moldavie, 1187 femmes contre 613 hommes d'Ukraine. 18. Taner Akpınar, op. cit., pp. 6-11. 
joué un rôle important dans l'augmentation du nombre de femmes migrantes choisissant la Turquie comme pays de destination, en facilitant la migration et la recherche d'emploi et d'hébergement. L'appel aux femmes migrantes dans certains secteurs a encouragé la création d'agences de recrutement, travaillant comme intermédiaires entre ces femmes et les employeurs ${ }^{19}$. Le nombre de ce type d'agences s'est très vite multi-

Le fait que les femmes turques préfèrent des emplois bénéficiant d'une protection sociale dans des secteurs mieux payés que le service domestique a indirectement accru la demande de femmes migrantes pour ce type d'emplois. plié et les femmes migrantes se sont trouvées obligées de leur payer une commission pour faciliter leur migration et leur recherche de travail ${ }^{20}$.

Enfin, la proximité géographique et, par conséquent, le bas coût des transports qui permettent d'effectuer des séjours au pays d'origine et, dans le cas de certains pays comme l'Azerbaïdjan, le Turkménistan et le Kazakhstan, la proximité historique, culturelle, linguistique ou ethnique, peuvent être considérées comme d'autres facteurs d'attraction de l'immigration en Turquie $^{21}$.

\section{Des emplois destinés aux femmes migrantes}

En Turquie, les services domestiques occupent la première place de la liste des emplois occupés par les femmes migrantes ${ }^{22}$. Comme on l'observe dans d'autres pays européens méditerranéens, dans les régions urbaines de la Turquie, l'affaiblissement des liens familiaux a allégé les tâches domestiques et facilité la garde des enfants. La participation des femmes turques au monde du travail a suscité une demande accrue d'aide aux tâches domestiques et de soin des personnes âgées ou malades ${ }^{23}$.
Le fait que les femmes turques préfèrent des emplois bénéficiant d'une protection sociale dans des secteurs mieux payés que le service domestique a indirectement accru la demande de femmes migrantes pour ce type d'emplois ${ }^{24}$. À partir du milieu des années 1990, les Bulgares, les Moldaves, suivies par les Arméniennes, les Géorgiennes, les Turkmènes, les Azerbaidjanaises, les Kirghizes ont formé la majorité des travailleuses étrangères des services du care et des services domestiques ${ }^{25}$.

Les métiers du tourisme et des loisirs s'adressent également aux femmes migrantes, notamment en raison du fait qu'elles connaissent une ou plusieurs langues étrangères et qu'elles acceptent de travailler sans protection sociale et avec des salaires plus faibles que les femmes turques ${ }^{26}$. Une partie considérable des femmes migrantes sont employées dans des agences de tourisme ou de transport, en tant que guides ou animatrices. Enfin, la Turquie est un pays de transit et de destination pour le trafic du sexe. La majorité des femmes et des jeunes filles visibles dans ce secteur sont originaires des pays de l'ex-URSS. Les ressortissantes de Moldavie, de Russie et de Turkménie forment environ $60 \%$ des femmes forcées à se livrer à la prostitution ${ }^{27}$. Développé au début des années 1990 dans les grandes villes de la Turquie, le travail sexuel constitue jusqu'à aujourd'hui l'un des secteurs qui recrutent le plus de femmes migrantes ${ }^{28}$.

\section{De l'exploitation aux mauvais traitements}

Les problèmes et les difficultés des femmes migrantes dépendent avant tout de leur statut migratoire. Les migrantes en situation irrégulière travaillant dans le secteur informel sont celles qui

19. Selmin Kaşka, "The new international migration and migrant women in Turkey: The case of Moldovan domestic workers", in Mirekoç Resaarch Projects, 2005-2006, p. 48. 20. Emel Akalın Ayşe, op. cit., p. 331. 21. Ahmet içduygu, Türkiye'de Kaçak Göç, 1stanbul Ticaret Odası Yayınları, nº 4, İstanbul, 2004, pp. 25-36. 22. Selmin Kaşka, "Ev içi Hizmetlerin Küreselleşmesi ve Türkiye’deki Göçmen Kadınlar", in Tes-Iş Dergisi, nº 3, 2005, p. 52. 23. Natalia Ribas Mateos, Véronique Manry (dir.), Mobilité au feminin. La place des fermmes dans le nouvel état au monde, Paris, Karthala, 2013, p. 386. 24. Sema Erder, op. cit., p. 200. 25. Emel Akalın Ayşe, "Ev işlerinde yeni emek çağı", in Express Dergisi, n 99, 2009. 26. Saniye Dedeoğlu, op. cit., 2011, p. 34. 27. International Organisation for Migration, Migration in Turkey, A Country Profile, 2008, p.34. http://www.turkey.iom.int/documents/migration_profile_turkey.pdf?entry 28. Ahmet içduygu, op. cit., 2004, p. 36. 
sont les vulnérables à toute forme d'exploitation ${ }^{29}$. Souvent dépendant d'un contrat conclu oralement entre l'employeur et la femme migrante, le travail domestique entraîne des conflits dans la fixation des horaires et des tâches à remplir ${ }^{30}$. L'exploitation par les employeurs est fréquemment observée dans le cas des femmes travaillant dans le soin des malades ou des personnes âgées: elles sont obligées par leurs employeurs d'assurer d'autres tâches domestiques qui ne font pas partie de leurs responsabilités ${ }^{31}$.

Parfois, les employeurs ou les agences d'emploi menacent les migrantes en situation irrégulière d'informer la police, confisquent leurs passeports, vont jusqu'à les forcer à travailler sans les payer (ou sans les payer régulièrement) ou les obligent à prendre part à des activités illégales comme le travail sexuel ${ }^{32}$. Leur situation irrégulière les empêche de porter plainte devant la police, de demander une protection juridique et induit un certain mode d'esclavage dans le travail ${ }^{33}$. À l'instar d'autres pays accueillant des femmes migrantes, même si le travail domestique assure une sécurité relative, l'exploitation et les mauvais traitements de la part des employeurs et des agences de recrutement ${ }^{34}$ favorisent la peur de l'expulsion et l'isolement ${ }^{35}$ des migrantes irrégulières.

Plusieurs femmes migrantes se disent victimes de violences, qu'elles soient verbales, économiques, physiques ou sexuelles ${ }^{36}$. Un des exemples les plus courants de la violence verbale est la catégorisation générale de ces femmes, notamment celles originaires de Russie et d'Ukraine, en tant que travailleurs du sexe. Dans la société turque, avec l'appui des médias, les femmes migrantes russes et ukrainiennes, mais également les femmes originaires de l'Europe de l'Est, appelées souvent "Natasha", sont considérées comme des travailleuses du sexe et des "prostituées qui font ce métier pour l'argent et pour leur propre plaisir, porteuses de maladies sexuellement transmissibles ${ }^{37 "}$. Cette situation s'observe dans d'autres pays. Cette catégorisation des migrantes originaires des pays de l'Europe orientale et de la Russie dans l'opinion publique encourage leur harcèlement quotidien ${ }^{38}$. La durée excessive du temps de travail et la charge de travail, la réduction du temps libre, la peur de l'expulsion, le manque de leur pays d'origine et de leur famille, particulièrement de leurs enfants, aggravent l'état de santé physique mais aussi psychique des femmes migrantes ${ }^{39}$. Certaines d'entre elles rencontrent des problèmes de santé qu'elles ne déclarent pas aux services concernés ou à leurs employeurs. Elles doivent, en effet, prendre en charge leurs frais médicaux ${ }^{40}$ et ont peur de perdre leur emploi.

\section{Conclusion}

En Turquie, la plupart des migrantes doivent faire face à toute une série de mauvais traitements, conséquences du travail illégal. Malgré les nouvelles réglementations dans la politique de l'immigration turque, incluant entre autres le règlement de la migration des employées domestiques, les immigrées irrégulières demeurent vulnérables. Cette vulnérabilité, qui tient à la place de la femme dans la société turque, est particulièrement liée, d'après nous, à l'offre accrue de l'économie informelle turque, tolérée voire encouragée par les politiques étatiques : depuis les années 1990, un système de double marché du travail s'est mis en place en Turquie en faveur de certains secteurs économiques. Malgré les violences subies, il permet à ces catégories spécifiques de main-d'œuvre étrangère d'avoir des revenus plus élevés que dans leur pays d'origine.

29. Davidson J. O'Connell, "New slavery, old binaries: human trafficking and the borders of 'freedom", in Global Networks, vol. 10, $n^{\circ}$ 2, 2010, p. 257. 30. Nilay Etiler, Kuvvet Lordoğlu, op. cit., 2010, p. 99. 31. Emel Akalın Ayşe, op. cit., p. 334. 32. Nilay Etiler, Kuvvet Lordoğlu, op. cit., p. 99. 33. Derya Demirdizen, op. cit., p. 340. 34. Nilay Etiler, Kuvvet Lordoğlu, op. cit., 2010, p. 99. 35. Emel Akalın Ayşe, op. cit., p. 327. 36. Ibid., p. 333. 37. Emel Coşkun, “Türkiye'de Göçmen Kadınlar ve Seks Ticareti”, in Çalışma ve Toplum, n³ 2014, p. 196. 38. Ayşen Üstübici, "Türkiye’ye yönelik Kadın Göçü: Seks işçileri ve Ev 1çi Hizmetlilerin Kişisel Güvenlik Sorunlarını 1lişkilendirmek", 2011, p. 5. Source : www.umut.org.tr,Hukukun Gencleri/TamMetinlerSunular/AysenUstubici.pdf 39. Ibid., p.110. 40. Nilay Etiler, Kuvvet Lordoğlu, op. cit., p. 108. 